\title{
Waves in Microstructured Conducting Sheath Helix Embedded Optical Guides with Chiral Nihility and Chiral Materials
}

\author{
N. Iqbal, M. A. Baqir, and P. K. Choudhury \\ Institute of Microengineering and Nanoelectronics, Universiti Kebangsaan Malaysia (UKM), 43600 Bangi, Selangor, Malaysia \\ Correspondence should be addressed to P. K. Choudhury; pankaj@ukm.edu.my
}

Received 10 April 2014; Revised 28 May 2014; Accepted 28 May 2014; Published 17 June 2014

Academic Editor: Christian Brosseau

Copyright (c) 2014 N. Iqbal et al. This is an open access article distributed under the Creative Commons Attribution License, which permits unrestricted use, distribution, and reproduction in any medium, provided the original work is properly cited.

\begin{abstract}
The paper deals with the sustainment of electromagnetic waves in circularly cylindrical optical guide with chiral nihility and chiral materials in the core and the clad sections, respectively. A perfectly conducting tightly wound helix is introduced at the coreclad interface. The eigenvalue relation for such a complex optical microstructured guide is deduced by applying suitable boundary conditions at the core-clad interface, and the dispersion behavior is analyzed by varying the pitch angle of helix. The sustainment of energy flux density in such optical guides is estimated under various structural conditions, and the density patterns in core-clad sections are anatomized analytically.
\end{abstract}

\section{Introduction}

Due to many exotic electromagnetic characteristics, chiral materials attracted the attention of investigators. The interesting property of chiral medium is the deficiency in uniformity of its inner structure, which consequently results in cross-coupling of electric and magnetic fields $[1,2]$, making thereby the analytical treatments rather formidable. One of the interesting properties of chiral metamaterials is having the phenomenon of negative refraction/reflection that can be achieved by increasing the chirality $[3,4]$, and the applications of such phenomenon are described in $[5,6]$ as well.

Investigations pivoted to wave propagation in various forms of chirowaveguides have appeared in the literature [7-10]. Within the context, chiral nihility metamaterials can be realized through chiral materials which, by definition, have approximately vanishing dielectric permittivity $\varepsilon$ and magnetic permeability $\mu$, but nonzero chirality parameter [11]. References $[12,13]$ discuss the concept of nihility with the medium having the permittivity and permeability simultaneously zero. Although the propagation of electromagnetic waves through nihility medium is not possible, in chiral nihility medium, similar to chiral mediums, two oppositely (left- and right-circularly) polarized waves propagate. One of the interesting features of chiral nihility medium remains as the propagation of backward waves [11].

Apart from the electromagnetic analyses of varieties of conventional chirowaveguides, investigations have been reported focusing on the dispersion characteristics and/or the power transmission patterns of various other new forms of waveguide structures embedded with chiral materials $[14,15]$. These constitute complex mediums, and the wave propagation through such structures would further be governed on demand by the use of twisted clad chiral guides [16]. These twists are in the form of a mounting of conducting sheath helix structure at the core-clad interface-the pitch angle of helix plays the determining role in tuning the dispersion features of the guide [17-20]. This concept is similar to the implementation of conducting helical structures in low- and medium-power travelling wave tubes [21] for the generation/amplification of microwaves.

Keeping in mind the concept of chiral nihility medium and the possible alternations of the dispersion characteristics of a fiber guiding structure, the present communication aims at the investigation of electromagnetic wave propagation in 


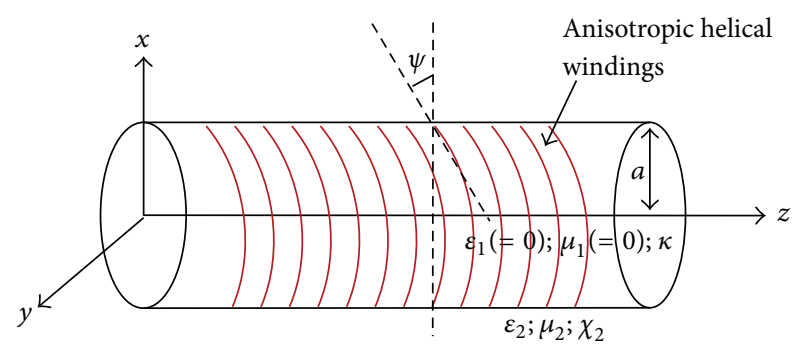

FIGURE 1: Schematic of the guide structure under consideration.

complex structured guide with chiral nihility core, which is cladded with chiral medium, and having introduced a conducting sheath helix structure at the core-clad interface. Considering the case of sheath helix with each turn being isolated from the neighboring one, but still the helical winding being continuous, the dispersion behavior of fiber is analyzed under the variation of the angle of pitch of conducting helical twists, which is followed with the determination of energy flux density patterns in the waveguide structure.

\section{Theory}

We consider an optical fiber guide with circular cross-section, the core of which is made of chiral nihility material, whereas the clad section is composed of chiral material. Also, the coreclad interface is loaded with a perfectly conducting sheath helix, which is inclined at an angle $\psi$ with respect to the vertical tangent drawn (upon a longitudinal view of guide) over the interface; Figure 1 illustrates the schematic of such a microstructured guide. The quantity $\psi$ is termed as the helix pitch angle, which actually exists at the core surface having a radius of $\rho=a$. The impedance of chiral nihility medium can be represented as $\eta_{1}=\lim _{\mu, \varepsilon \rightarrow 0} \sqrt{(\mu / \varepsilon)}$. The chiral clad is assumed to have the admittance $\chi_{2}$ and is nonmagnetic in nature; that is, the magnetic permeability for this region can be written as $\mu_{2}=\mu_{0}, \mu_{0}$ being the free-space permeability.

Taking into account the wave propagation to be harmonic in time $t$ and the $z$-axis (the optical axis of guide), we may write the constitutive relations for the mediums, which the guide is composed of. Within the context, the constitutive relations for isotropic chiral medium for a time harmonic field are given as [11]

$$
\begin{aligned}
& \mathbf{D}_{1}=\varepsilon_{1} \mathbf{E}_{1}-j \kappa \sqrt{\mu_{0} \varepsilon_{0}} \mathbf{H}_{1}, \\
& \mathbf{B}_{1}=\mu_{1} \mathbf{H}_{1}+j \kappa \sqrt{\mu_{0} \varepsilon_{0}} \mathbf{E}_{\mathbf{1}},
\end{aligned}
$$

where $\varepsilon_{1}, \mu_{1}$, and $\kappa$ are, respectively, permittivity, permeability, and chirality parameter of chiral medium. For a chiral nihility medium, we assume the permittivity and the permeability both to be zero at certain frequency, but the chirality remains nonzero. As such, (1a) and (1b) can be written for chiral nihility medium as [11]

$$
\begin{aligned}
\mathbf{D}_{1} & =-j \kappa \sqrt{\mu_{0} \varepsilon_{0}} \mathbf{H}_{1}, \\
\mathbf{B}_{1} & =j \kappa \sqrt{\mu_{0} \varepsilon_{0}} \mathbf{E}_{\mathbf{1}} .
\end{aligned}
$$

Implementing Maxwell's equations and using the above mentioned constitutive relations, we ultimately obtain the components of electric/magnetic fields in the core section as [22]

$$
E_{\rho 1}=(A+B)\left[\frac{j m \kappa k_{0}}{k_{\rho}^{2} \rho} J_{m}\left(k_{\rho} \rho\right)-\frac{j \beta}{k_{\rho}} J_{m}^{\prime}\left(k_{\rho} \rho\right)\right] e^{j m \varphi},
$$

$$
H_{\rho 1}=\frac{j}{\eta_{1}}(A-B)\left[\frac{j m \kappa k_{0}}{k_{\rho}^{2} \rho} J_{m}\left(k_{\rho} \rho\right)-\frac{j \beta}{k_{\rho}} J_{m}^{\prime}\left(k_{\rho} \rho\right)\right] e^{j m \varphi},
$$

$$
\begin{aligned}
E_{\varphi 1} & =(A+B)\left[\frac{m \beta}{k_{\rho}^{2} \rho} J_{m}\left(k_{\rho} \rho\right)-\frac{\kappa k_{0}}{k_{\rho}} J_{m}^{\prime}\left(k_{\rho} \rho\right)\right] e^{j m \varphi}, \\
H_{\varphi 1} & =\frac{j}{\eta_{1}}(A-B)\left[\frac{m \beta}{k_{\rho}^{2} \rho} J_{m}\left(k_{\rho} \rho\right)-\frac{\kappa k_{0}}{k_{\rho}} J_{m}^{\prime}\left(k_{\rho} \rho\right)\right] e^{j m \varphi},
\end{aligned}
$$

$$
\begin{aligned}
E_{z 1} & =(A+B) J_{m}\left(k_{\rho} \rho\right) e^{j m \varphi}, \\
H_{z 1} & =\frac{j}{\eta_{1}}(A-B) J_{m}\left(k_{\rho} \rho\right) e^{j m \varphi} .
\end{aligned}
$$

In all the equations above, the subscript 1 corresponds to the situations in fiber guide core. In (3a), (3b), (3c), (3d), (3e), and (3f), $A$ and $B$ are unknown constants, $\eta_{1}=\lim _{\mu, \varepsilon \rightarrow 0} \sqrt{(\mu / \varepsilon)}$ is impedance of chiral nihility medium, $J_{m}(\cdot)$ is Bessel function of the first kind (with prime representing the first-order derivative with respect to the argument), $m$ is an integer representing the order of mode, and $k_{\rho \pm}=\left(k_{ \pm}^{2}-\beta^{2}\right)^{1 / 2}=$ $k_{0} \sqrt{\kappa^{2}-\left(\beta / k_{0}\right)^{2}}=k_{\rho}$ are transverse wave numbers of right- and left-circularly polarized eigenwaves in core. For the propagating mode, $k_{\rho}$ must be real valued; an imaginary value of $k_{\rho}$ will correspond to nonpropagating mode.

Now, the constitutive relations for homogeneous, lossless, and isotropic chiral mediums are given as [2]

$$
\begin{aligned}
& \mathbf{D}_{2}=\varepsilon_{2} \mathbf{E}_{2}-j \chi_{2} \mathbf{B}_{2}, \\
& \mathbf{H}_{2}=-j \chi_{2} \mathbf{E}_{2}+\frac{\mathbf{B}_{2}}{\boldsymbol{\mu}_{2}},
\end{aligned}
$$

with $\varepsilon_{2}$ as the electric permittivity of chiral medium, and the other parameters are defined before. As the region is being composed of isotropic material, the electric permittivity $\varepsilon_{2}$ is taken to be a constant.

The use of Maxwell's equations and the constitutive relations in $(4 \mathrm{a})$ and $(4 \mathrm{~b})$ provides the following expressions for electric/magnetic fields in the clad section of guide [2]:

$$
\begin{aligned}
E_{\rho 2}= & j \eta_{c 2} C\left[\tau_{2+} \beta K_{m}^{\prime}\left(\tau_{2+} \rho\right)-\frac{m \Omega_{2+}}{\rho \eta_{c 2} \tau_{2-}^{2}} K_{m}\left(\tau_{2+} \rho\right)\right] e^{j m \varphi} \\
& +D\left[\tau_{2-} \beta K_{m}^{\prime}\left(\tau_{2-} \rho\right)-\frac{m \Omega_{2-}}{\rho \eta_{c 2} \tau_{2+}^{2}} K_{m}\left(\tau_{2-} \rho\right)\right] e^{j m \varphi},
\end{aligned}
$$




$$
\begin{aligned}
H_{\rho 2}= & j C\left[\tau_{2+} \beta K_{m}^{\prime}\left(\tau_{2+} \rho\right)-\frac{m \Omega_{2+}}{\rho \eta_{c 2} \tau_{2-}^{2}} K_{m}\left(\tau_{2+} \rho\right)\right] e^{j m \varphi} \\
& -D\left[\tau_{2-} \beta K_{m}^{\prime}\left(\tau_{2-} \rho\right)-\frac{m \Omega_{2-}}{\rho \eta_{c 2} \tau_{2-}^{2}} K_{m}\left(\tau_{2-} \rho\right)\right] e^{j m \varphi}, \\
E_{\varphi 2}= & j \eta_{c 2} C\left[\frac{m \beta}{\rho} K_{m}\left(\tau_{2+} \rho\right)-\frac{\Omega_{2+} \tau_{2+}}{\eta_{c 2} \tau_{2-}^{2}} K_{m}^{\prime}\left(\tau_{2+} \rho\right)\right] e^{j m \varphi} \\
+ & j \eta_{c 2} D\left[\frac{m \beta}{\rho} K_{m}\left(\tau_{2-} \rho\right)-\frac{\Omega_{2-} \tau_{2-}}{\eta_{c 2} \tau_{2+}^{2}} K_{m}^{\prime}\left(\tau_{2-} \rho\right)\right] e^{j m \varphi}, \\
H_{\varphi 2}= & C\left[\frac{m \beta}{\rho} K_{m}\left(\tau_{2+} \rho\right)-\frac{\Omega_{2+} \tau_{2+}}{\eta_{c 2} \tau_{2-}^{2}} K_{m}^{\prime}\left(\tau_{2+} \rho\right)\right] e^{j m \varphi} \\
& +D\left[\frac{m \beta}{\rho} K_{m}\left(\tau_{2-} \rho\right)-\frac{\Omega_{2-} \tau_{2-}}{\eta_{c 2} \tau_{2+}^{2}} K_{m}^{\prime}\left(\tau_{2-} \rho\right)\right] e^{j m \varphi}, \\
E_{z 2}= & j \eta_{c 2}\left[\tau_{2+}^{2} C K_{m}\left(\tau_{2+} \rho\right)-\tau_{2-}^{2} D K_{m}\left(\tau_{2-} \rho\right)\right] e^{j m \varphi}, \quad(5 \mathrm{e}) \\
H_{z 2}= & {\left[\tau_{2+}^{2} C K_{m}\left(\tau_{2+} \rho\right)+\tau_{2-}^{2} D K_{m}\left(\tau_{2-} \rho\right)\right] e^{j m \varphi} . }
\end{aligned}
$$

In (4a), (4b), (5a), (5b), (5c), (5d), (5e), and (5f), the subscript 2 represents the situations in clad section. Further, in (5a), (5b), (5c), (5d), (5e), and (5f), $C$ and $D$ are unknown constants, and $K_{m}(\cdot)$ is the modified Bessel function (with prime representing the differentiation with respect to the argument). Also, the other terms used in these equations have meanings as follows [15]:

$$
\begin{gathered}
\tau_{2 \pm}=\left[\beta^{2}-\gamma_{2}^{2}-\left(2 \omega^{2} \mu_{0}^{2} \chi_{2}^{2} \pm \frac{2 \omega^{2} \mu_{0}^{2} \chi_{2}}{\eta_{c 2}}\right)\right]^{1 / 2}, \\
\Omega_{2 \pm}=\omega \mu_{0}\left[\chi_{2} \eta_{c 2}\left(\gamma_{2}^{2}+\beta^{2}\right) \pm\left(\beta^{2}-\gamma_{2}^{2}\right)\right] \\
\gamma_{2}=\left(\omega^{2} \varepsilon_{2} \mu_{0}\right)^{1 / 2} \\
\eta_{c 2}=\left(\frac{\eta_{2}^{2}}{1+\eta_{2}^{2} \chi_{2}^{2}}\right)^{1 / 2}, \\
\eta_{2}=\left(\frac{\mu_{0}}{\varepsilon_{2}}\right)^{1 / 2} .
\end{gathered}
$$

Now, by using the above derived field equations, and applying the boundary conditions [18] at the layer interface of microstructured guide of Figure 1, after some mathematical steps, we obtain the characteristic eigenvalue equation for the guiding structure, as follows:

$$
\begin{gathered}
\frac{2 j \eta_{1} \eta_{c 2}^{2}}{k_{\rho}^{4}}\left[\left\{\frac{\beta m}{a} J_{m}\left(k_{\rho} a\right) \cos \psi-\kappa k_{0} k_{\rho} J_{m}^{\prime}\left(k_{\rho} a\right) \cos \psi\right.\right. \\
\left.+k_{\rho}^{2} J_{m}\left(k_{\rho} a\right) \sin \psi\right\}^{2}
\end{gathered}
$$

$$
\begin{aligned}
& \times\left\{\frac{\Omega_{2+} \tau_{2+}}{\eta_{2 c}} K_{m}^{\prime}\left(\tau_{2+} a\right) K_{m}\left(\tau_{2-} a\right)\left(\cos ^{2} \psi-\sin ^{2} \psi\right)\right. \\
&+\frac{\beta m \tau_{2-}^{2}}{a} K_{m}\left(\tau_{2+} a\right) K_{m}\left(\tau_{2-} a\right)\left(\sin ^{2} \psi-\cos ^{2} \psi\right) \\
&-\frac{\Omega_{2-} \tau_{2-}}{\eta_{c 2}} K_{m}\left(\tau_{2+} a\right) K_{m}^{\prime}\left(\tau_{2-} a\right) \\
&+\frac{\beta m \tau_{2+}^{2}}{a} K_{m}\left(\tau_{2+} a\right) K_{m}\left(\tau_{2-} a\right) \\
&\left.\left.-2 \tau_{2+}^{2} \tau_{2-}^{2} K_{m}\left(\tau_{2+} a\right) K_{m}\left(\tau_{2-} a\right) \sin \psi \cos \psi\right\}\right] \\
&=f(\beta)(\text { say })=0 .
\end{aligned}
$$

The use of field expressions corresponding to the core and the clad sections of microstructured guide, as represented in (3a), (3b), (3c), (3d), (3e), and (3f) and (5a), (5b), (5c), $(5 \mathrm{~d}),(5 \mathrm{e})$, and $(5 \mathrm{f})$, respectively, would provide the energy flux densities [23] in the two sections of guide. After some mathematical steps, it can be shown that the flux densities in the core $\left(S_{z 1}\right)$ and the clad $\left(S_{z 2}\right)$ sections will assume the expressions, as follows:

$$
\begin{aligned}
S_{z 1}= & \frac{|A|^{2}-|B|^{2}}{4 \eta_{1} k_{\rho}^{2}}\left[\left(\kappa k_{0}+\beta\right)^{2}\left\{J_{m+1}\left(k_{\rho} \rho\right)\right\}^{2}\right. \\
S_{z 2}= & \frac{D^{2}}{2}\left\{\tau_{2-} \beta K_{m}^{\prime}\left(\tau_{2-} \rho\right)-\frac{m \Omega_{2-}}{\rho \eta_{c 2} \tau_{2+}^{2}} K_{m}\left(\tau_{2+} \rho\right)\right\} \\
& \times\left\{\frac{\beta m}{\rho} K_{m}\left(\tau_{2-} \rho\right)-\frac{\Omega_{2-} \tau_{2-}}{\eta_{c 2} \tau_{2+}^{2}} K_{m}^{\prime}\left(\tau_{2+} \rho\right)\right\} \\
& +\frac{C D}{2}\left\{\tau_{2-} \beta K_{m}^{\prime}\left(\tau_{2-} \rho\right)-\frac{m \Omega_{2-}}{\rho \eta_{c 2} \tau_{2+}^{2}} K_{m}\left(\tau_{2+} \rho\right)\right\} \\
& \times\left\{\frac{\beta m}{\rho} K_{m}\left(\tau_{2+} \rho\right)-\frac{\Omega_{2+} \tau_{2+}}{\eta_{c 2} \tau_{2-}^{2}} K_{m}^{\prime}\left(\tau_{2+} \rho\right)\right\} \\
& +\frac{C^{2} \eta_{c 2}}{2}\left\{\frac{\beta m}{\rho} K_{m}\left(\tau_{2+} \rho\right)-\frac{\Omega_{2+} \tau_{2+}}{\eta_{c 2} \tau_{2-}^{2}} K_{m}^{\prime}\left(\tau_{2+} \rho\right)\right\} \\
& \times\left\{\tau_{2+} \beta K_{m}^{\prime}\left(\tau_{2-} \rho\right)-\frac{m \Omega_{2+}}{\rho \eta_{c 2} \tau_{2-}^{2}} K_{m}\left(\tau_{2+} \rho\right)\right\} \\
& -\frac{C D \eta_{c 2}}{2}\left\{\frac{\beta m}{\rho} K_{m}\left(\tau_{2-} \rho\right)-\frac{\Omega_{2-} \tau_{2-}}{\eta_{c 2} \tau_{2+}^{2}} K_{m}^{\prime}\left(\tau_{2-} \rho\right)\right\} \\
& \times\left\{\tau_{2+} \beta K_{m}^{\prime}\left(\tau_{2+} \rho\right)-\frac{m \Omega_{2+}}{\rho \eta_{c 2} \tau_{2-}^{2}} K_{m}\left(\tau_{2+} \rho\right)\right\}
\end{aligned}
$$

Equations (8a) and (8b) contain the unknown constants $A$, $B, C$, and $D$. However, the values of these constants are to 
be determined in terms of only one constant so that the flux densities can be normalized. This essentially needs the use of boundary conditions. After implementing those, we finally obtain the values of constants $B, C$, and $D$ in terms of the constant $A$, as follows:

$$
\begin{aligned}
& B=\frac{\alpha_{1}\left(\alpha_{2} \alpha_{3}+\alpha_{4} \alpha_{5}\right)-2 \alpha_{3} \alpha_{5} \alpha_{6}}{\alpha_{1}\left(\alpha_{2} \alpha_{3}+\alpha_{4} \alpha_{5}\right)+2 \alpha_{3} \alpha_{5} \alpha_{6}} A \\
& C=\frac{-\alpha_{6} \alpha_{3}}{\alpha_{2} \alpha_{3}+\alpha_{4} \alpha_{5}}\left\{\frac{2 \alpha_{1}\left(\alpha_{2} \alpha_{3}+\alpha_{4} \alpha_{5}\right)}{\alpha_{1}\left(\alpha_{2} \alpha_{3}+\alpha_{4} \alpha_{5}\right)+2 \alpha_{3} \alpha_{5} \alpha_{6}}\right\} A \\
& D=\frac{-\alpha_{6} \alpha_{5}}{\alpha_{2} \alpha_{3}+\alpha_{4} \alpha_{5}}\left\{\frac{2 \alpha_{1}\left(\alpha_{2} \alpha_{3}+\alpha_{4} \alpha_{5}\right)}{\alpha_{1}\left(\alpha_{2} \alpha_{3}+\alpha_{4} \alpha_{5}\right)+2 \alpha_{3} \alpha_{5} \alpha_{6}}\right\} A .
\end{aligned}
$$

In (9a), (9b), and (9c) the used symbols have meanings, as follows:

$$
\begin{aligned}
\alpha_{1}= & \frac{j}{\eta_{1}} J_{m}\left(k_{\rho} a\right) \sin \psi \\
& +\frac{j}{\eta_{1}}\left\{\frac{\beta m}{a k_{\rho}^{2}} J_{m}\left(k_{\rho} a\right)-\frac{\kappa k_{0}}{k_{\rho}} J_{m}^{\prime}\left(k_{\rho} a\right)\right\} \cos \psi \\
\alpha_{2}= & j \eta_{c 2} \tau_{2+}^{2} K_{m}\left(\tau_{2+} a\right) \cos \psi \\
& -j \eta_{c 2}\left\{\frac{\beta m}{a} K_{m}\left(\tau_{2+} a\right)-\frac{\Omega_{2+} \tau_{2+}}{\eta_{c 2} \tau_{2-}^{2}} K_{m}^{\prime}\left(\tau_{2+} a\right)\right\} \sin \psi \\
\alpha_{3}= & \tau_{2-}^{2} K_{m}\left(\tau_{2-} a\right) \sin \psi \\
& +\left\{\frac{\beta m}{a} K_{m}\left(\tau_{2-} a\right)-\frac{\Omega_{2-} \tau_{2-}}{\eta_{c 2} \tau_{2+}^{2}} K_{m}^{\prime}\left(\tau_{2-} a\right)\right\} \cos \psi \\
\alpha_{6}=J_{m} & \left(k_{\rho} a\right) \cos \psi-\left\{\frac{\beta m}{a k_{\rho}^{2}} J_{m}\left(k_{\rho} a\right)-\frac{\kappa k_{0}}{k_{\rho}} J_{m}^{\prime}\left(k_{\rho} a\right)\right\} \sin \psi \\
\alpha_{5}= & \tau_{2+}^{2} K_{m}\left(\tau_{2+} a\right) \sin \psi \\
\alpha_{4}= & j \eta_{c 2} \tau_{2-}^{2} K_{m}\left(\tau_{2-} a\right) \cos \psi \\
& +j \eta_{c 2}\left\{\frac{\beta m}{a} K_{m}\left(\tau_{2-} a\right)-\frac{\Omega_{2-} \tau_{2-}}{\eta_{c 2} \tau_{2+}^{2}} K_{m}^{\prime}\left(\tau_{2-} a\right)\right\} \sin \psi
\end{aligned}
$$

The above derived equation (7) can be used to determine the modal behavior in the guide, and ( $8 \mathrm{a})$ and $(8 \mathrm{~b})$ will determine the flux density patterns therein.

\section{Results and Discussion}

In order to obtain the dispersion behavior of fiber guide, we consider the regions (of guide) as nonmagnetic in nature
TABLE 1: Propagation constants corresponding to particular modes.

\begin{tabular}{cccc}
\hline Core radius $a$ & Pitch angle $\psi$ & Propagation modes & $\beta\left(\mathrm{m}^{-1}\right)$ \\
\hline \multirow{4}{*}{$10 \mu \mathrm{m}$} & $0^{\circ}$ & $\mathrm{EH}_{01}$ & $5.7158 \times 10^{6}$ \\
& & $\mathrm{EH}_{11}$ & $5.8545 \times 10^{6}$ \\
& & $\mathrm{EH}_{-11}$ & $5.7958 \times 10^{6}$ \\
& $90^{\circ}$ & $\mathrm{EH}_{01}$ & $5.7458 \times 10^{6}$ \\
& $\mathrm{EH}_{11}$ & $5.8374 \times 10^{6}$ \\
& $\mathrm{EH}_{-11}$ & $5.8071 \times 10^{6}$ \\
\hline \multirow{3}{*}{$30 \mu \mathrm{m}$} & $\mathrm{EH}_{01}$ & $5.7898 \times 10^{6}$ \\
& & $\mathrm{EH}_{11}$ & $5.8149 \times 10^{6}$ \\
& & $\mathrm{EH}_{-11}$ & $5.7378 \times 10^{6}$ \\
& & $\mathrm{EH}_{01}$ & $5.7585 \times 10^{6}$ \\
& & $\mathrm{EH}_{11}$ & $5.8551 \times 10^{6}$ \\
& & $\mathrm{EH}_{-11}$ & $5.7955 \times 10^{6}$ \\
\hline
\end{tabular}

and the operating wavelength to be $1.55 \mu \mathrm{m}$. Further, the chirality of nihility core is assumed to be $\kappa=1.55$, and the refractive index and the chirality impedance of clad region are taken to be $n_{2}=1.54$ and $\chi_{2}=1.7 \times 10^{-3} \Omega^{-1}$, respectively, and the impedance of nihility core is taken to be as that of the free-space. As such, it remains explicit that we take into account the dielectric permittivity of the clad region to be a constant. Furthermore, we assume two different values of guide core radius, namely, $10 \mu \mathrm{m}$ and $30 \mu \mathrm{m}$, and the clad section is assumed to be infinitely extended. We first focus on the evaluation of the propagation constants corresponding to the low-order hybrid modes in the guide using two extreme values of the angle of pitch, that is, $0^{\circ}$ and $90^{\circ}$. Thereafter, the investigations are made of the flux density patterns corresponding to those modes as sustained in the guiding structure.

In order to obtain the propagation constants for the $\mathrm{EH}_{01}$, $\mathrm{EH}_{11}$, and $\mathrm{EH}_{-11}$ modes, we solve the dispersion relation as stated in (7) for the range of $\beta$. The corresponding plots are, however, not incorporated into the text. Table 1 provides the finally evaluated values of the propagation constants corresponding to the low-order hybrid modes when the fiber core radius values are taken as $10 \mu \mathrm{m}$ and $30 \mu \mathrm{m}$ under the two extreme values of helix pitch angle twists (i.e., $0^{\circ}$ and $90^{\circ}$ ).

Figures 2(a) and 2(b) illustrate the dispersion behavior of guiding structure corresponding to the pitch angle values as $0^{\circ}$ and $90^{\circ}$, respectively, considering the low-order modes belonging to $m=0,1$ and -1 . In these plots, the effective refractive index values $n_{\text {eff }}$ are plotted against the normalized frequency parameter $V$ [23]. In Figure 2(a), which corresponds to the situation when the helix pitch angle is oriented perpendicular to the direction of wave propagation, we observe that the effective refractive index corresponding to the $m=0$ mode shows a gradual decrease with increasing value of $V$. But, under the same situation, the modes for $m=1$ and $m=-1$ exhibit small increase in the effective refractive index values with the increase in $V$. Furthermore, for $m=1$ and $m=-1, n_{\text {eff }}$ remains almost similar throughout increasing $V$, which exhibits a kind of mode degeneracy in this case. 


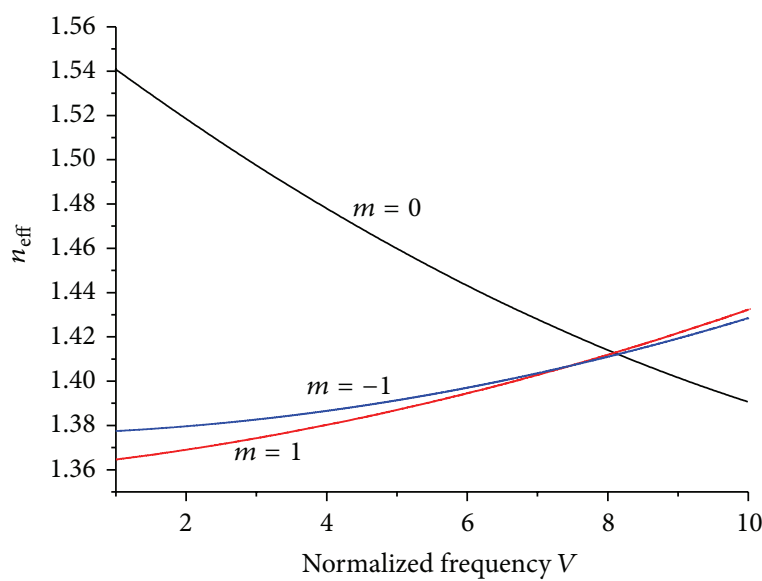

(a)

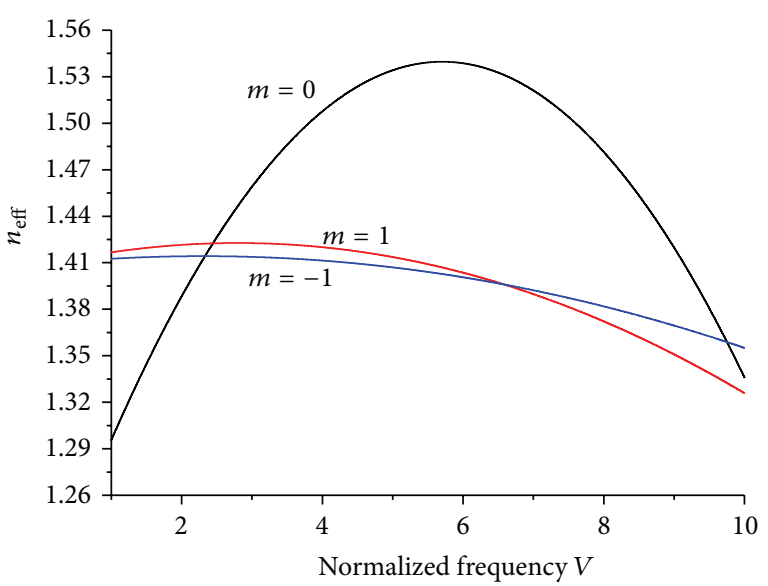

(b)

FIGURE 2: (a) Dispersion behavior of guiding structure corresponding to the helix pitch $\psi=0^{\circ}$. (b) Dispersion behavior of guiding structure corresponding to the helix pitch $\psi=90^{\circ}$.

Figure 2(b) corresponds to the situation when the helical wraps are oriented parallel to the direction of wave propagation, that is, $\psi=90^{\circ}$. We observe that the dispersion behavior is drastically altered in this case, and for all the values of azimuthal mode index $m$ (i.e., $m=0,1$ and - 1), the effective refractive index values show an initial rise with increasing $V$ followed by a decrease in their values for further increase in $V$. Particularly, corresponding to $m=0$, the dispersion behavior seems to be very different as $n_{\text {eff }}$ exhibits large variation with changing $V$. However, similar to the case of Figure 2(a), the situation of $\psi=90^{\circ}$ too brings in the circumstance of mode degeneracy corresponding to $m=1$ and $m=-1$ as a very small difference in $n_{\text {eff }}$ values can be observed with the increase in $V$ for these two mode orders. Comparing Figures 2(a) and 2(b), we further observe that the effective refractive index generally remains the maximum corresponding to the mode order $m=0$.

Figures 3 and 4, respectively, show the normalized flux density characteristics, as plotted against the radial variation (i.e., the guide radius), corresponding to core radius values as $10 \mu \mathrm{m}$ and $30 \mu \mathrm{m}$. In both the figures, plots of the flux patterns are shown for the sustained low-order hybrid $\mathrm{EH}_{01}, \mathrm{EH}_{11}$, and $\mathrm{EH}_{-11}$ modes by the solid, dashed, and dashed-dot lines. In order to plot these, the modal propagation constants are used, as enlisted in Table 1. We notice that these patterns have, in general, fluctuating kind of characteristics and exhibit dissipative trend as the peripheral region of core section is reached.

Figure 3(a) corresponds to the situation when the core radius of fiber guide is $10 \mu \mathrm{m}$ and the pitch angle of helical turns attains a $0^{\circ}$ value; that is, the orientation of conducting wraps is just perpendicular to the direction of wave propagation. We observe that, in this case, the $\mathrm{EH}_{01}$ mode occupies the highest flux density through the core region; it remains the maximum near the central section of guide and goes on decreasing as the core boundary is reached. Corresponding to the $\mathrm{EH}_{11}$ mode, flux is reduced as compared to that occupied by the $\mathrm{EH}_{01}$ mode, and also, the density maxima for the $\mathrm{EH}_{11}$ mode are slightly radially shifted outward in comparison with that of the $\mathrm{EH}_{01}$ mode. At the central region of guide, the flux maxima remains corresponding to the $\mathrm{EH}_{-11}$ mode, though it bears the least intensity as compared to the $\mathrm{EH}_{01}$ and $\mathrm{EH}_{11}$ modes. For all the modes, flux density gets damped as the boundary of the core section is reached.

Under similar dimensional and operating features of guide, a change in helix pitch angle to $90^{\circ}$ brings in substantial change in the intensities of flux density corresponding to all the sustained modes under consideration, which is very much evident from Figure 3(b). We find that the intensities are much increased in this case as compared to the situation of a $0^{\circ}$ helix pitch angle (Figure $3(\mathrm{a})$ ), and the $\mathrm{EH}_{01}$ mode still occupies the maximum amount of energy density. The field maxima corresponding to the $\mathrm{EH}_{-11}$ mode remain in the central region of core section-the feature as observed in Figure 3(b). For all the modes, similar to as seen in Figure 3(a), the energy density goes on dissipating as the peripheral region of core section is reached.

Flux density pattern results corresponding to an increased dimension of guide are shown in Figure 4, wherein the core section of the guide is assumed to have a radius as $30 \mu \mathrm{m}$. Keeping the other operating conditions unchanged, we consider the values of helix pitch angle as $0^{\circ}$ and $90^{\circ}$, and the plots of the flux density patterns are illustrated in Figures 4(a) and 4(b), respectively. We observe in Figure 4(a) that, for a $0^{\circ}$ helix pitch, the flux density patterns corresponding to all the modes taken into account exhibit dissipating ripples, but the overall intensities are much higher than that observed in Figure 3(a). This is essentially due to the increased dimension of the core region in this case. However, the maximum density of flux is occupied by the $\mathrm{EH}_{01}$ mode, similar to what is noticed before for the guiding structure with lower dimension (Figure 3(a)). Also, the density maxima at the central section of guide correspond to the $\mathrm{EH}_{-11}$ mode.

For a $90^{\circ}$ helix pitch angle, we observe from Figure 4(b) that the patterns of flux densities are very much similar to the case of $0^{\circ}$ pitch (Figure $4(\mathrm{a})$ ), except the fact that 


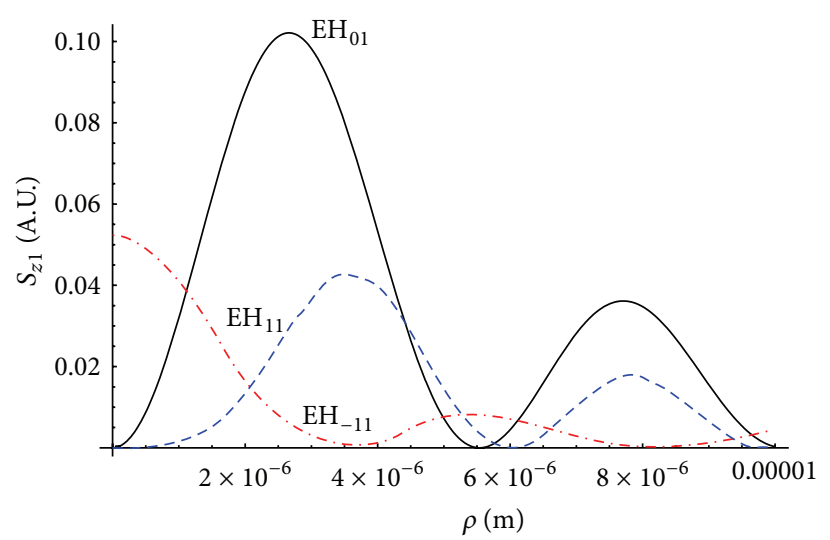

(a)

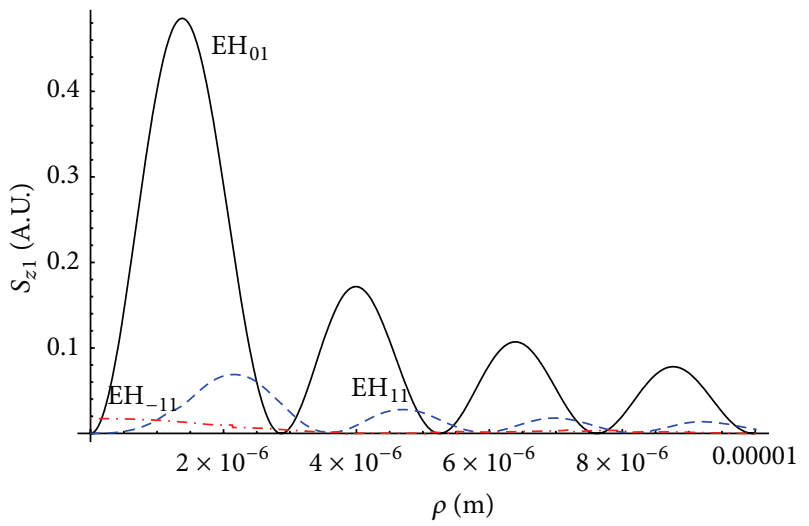

(b)

FIGURE 3: (a) Energy flux density in the core section corresponding to $\mathrm{EH}_{01}$ (solid line), $\mathrm{EH}_{11}$ (dashed line), and $\mathrm{EH}_{-11}$ (dashed-dot line) modes under the situation $a=10 \mu \mathrm{m}$ and $\psi=0^{\circ}$. (b) Energy flux density in the core section corresponding to $\mathrm{EH}_{01}$ (solid line), $\mathrm{EH}_{11}$ (dashed line), and $\mathrm{EH}_{-11}$ (dashed-dot line) modes under the situation $a=10 \mu \mathrm{m}$ and $\psi=90^{\circ}$.

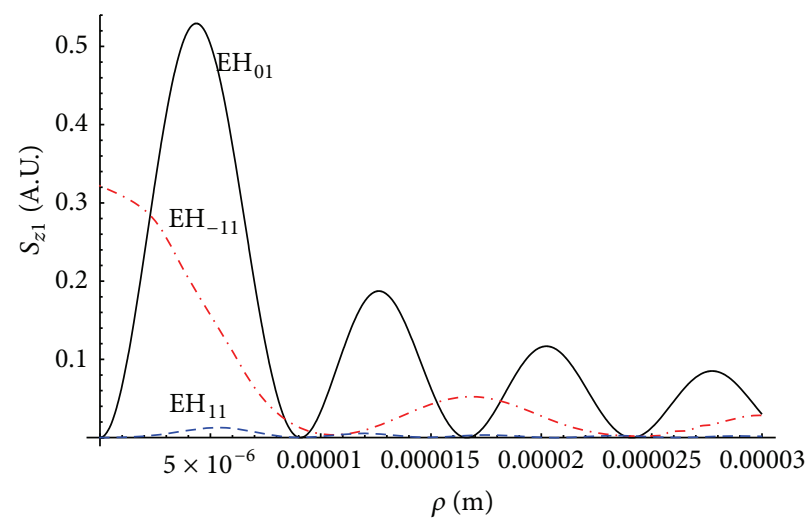

(a)

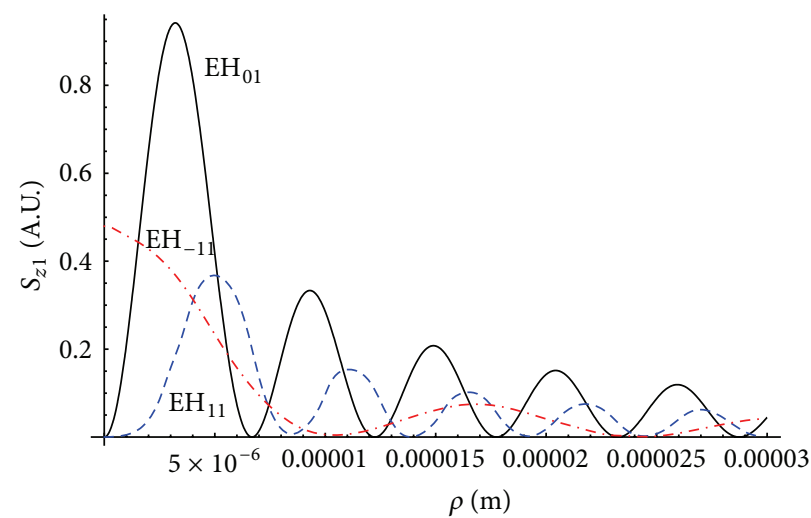

(b)

Figure 4: (a) Energy flux density in the core section corresponding to $\mathrm{EH}_{01}$ (solid line), $\mathrm{EH}_{11}$ (dashed line), and $\mathrm{EH}_{-11}$ (dashed-dot line) modes under the situation $a=30 \mu \mathrm{m}$ and $\psi=0^{\circ}$. (b) Energy flux density in the core section corresponding to $\mathrm{EH}_{01}$ (solid line), EH $\mathrm{EH}_{11}(\mathrm{dashed}$ line), and $\mathrm{EH}_{-11}$ (dashed-dot line) modes under the situation $a=30 \mu \mathrm{m}$ and $\psi=90^{\circ}$.

the magnitudes are slightly increased in this case, and the density characteristic exhibits more dissipating ripples. As such, the increase of fiber dimension makes the effect due to the alteration of pitch angle lesser; guides with smaller dimension will be more effective to the variation of embedded helix pitch; this becomes quite evident when Figures 3(a) and 4(a) are observed together (a closer comparative look at Figures 3(b) and 4(b) too reveals similar aspect).

\section{Conclusion}

The aforementioned investigation reveals that the helix pitch angle greatly governs the wave propagation features of guides composed of the mixture of chiral nihility and chiral materials. Considering the low-order hybrid modes, the respective propagations constants are evaluated followed with the investigations in respect of dispersion properties and flux density patterns taking into account the variations in guide dimension as well as helix pitch angle. It has been found that the dispersion features of the guide are very much altered upon a change in helix pitch. The flux density patterns reveal that energy in the core section remains the maximum corresponding to the $\mathrm{EH}_{01}$ mode. Also, the density patterns have ripples, and these go on decreasing as the core boundary of the guide is reached. Furthermore, the flux density gets amplified upon changing the pitch angle from $0^{\circ}$ to $90^{\circ}$. This feature of such guides made of chiral nihility and/or chiral materials may find varieties of applications in optics industries.

\section{Conflict of Interests}

The authors declare that there is no conflict of interests regarding the publication of this paper.

\section{Acknowledgments}

The authors are thankful to the Ministry of Higher Education (Malaysia) for financially supporting the work. Also, the 
authors gratefully acknowledge the constructive criticisms made by the two anonymous reviewers, which greatly helped to improve the content of paper.

\section{References}

[1] S. Bassiri, C. H. Papas, and N. Engheta, "Electromagnetic wave propagation through a dielectric-chiral slab interface and through a chiral slab," Journal of the Optical Society of America A, vol. 5, no. 9, pp. 1450-1459, 1988.

[2] A. K. Singh, S. Kh. Singh, P. Khastgir, S. P. Ojha, and O. N. Singh, "Modal cutoff condition of an optical chiral fiber with different chiralities in the core and the cladding," Journal of the Optical Society of America B, vol. 11, pp. 1283-1287, 1994.

[3] C. Monzon and D. W. Forester, "Negative refraction and focusing of circularly polarized waves in optically active media," Physical Review Letters, vol. 95, pp. 123904.1-123904.4, 2005.

[4] Z. Li, K. B. Alici, H. Caglayan, M. Kafesaki, C. M. Soukoulis, and E. Ozbay, "Composite chiral metamaterials with negative refractive index and high values of the figure of merit," Optics Express, vol. 20, no. 6, pp. 6146-6156, 2012.

[5] S. Tretyakov, A. Sihvola, and L. Jylhä, "Backward-wave regime and negative refraction in chiral composites," Photonics and Nanostructures: Fundamentals and Applications, vol. 3, no. 2-3, pp. 107-115, 2005.

[6] C.-W. Qiu, H.-Y. Yao, L.-W. Li, S. Zouhdi, and T.-S. Yeo, "Backward waves in magnetoelectrically chiral media: propagation, impedance, and negative refraction," Physical Review B: Condensed Matter and Materials Physics, vol. 75, no. 15, Article ID 155120, 2007.

[7] A. Lakhtakia, "A mini review on isotropic chiral mediums," in Electromagnetic Fields In Unconventional Materials and Structures, O. N. Singh and A. Lakhtakia, Eds., pp. 125-149, Wiley, New York, NY, USA, 2000.

[8] A. Lakhtakia, "Enhancement of optical activity of chiral sculptured thin films by suitable infiltration of void regions," Optik, vol. 112, no. 4, pp. 145-148, 2001.

[9] A. Nair and P. K. Choudhury, "On the analysis of field patterns in chirofibers," Journal of Electromagnetic Waves and Applications, vol. 21, no. 15, pp. 2277-2286, 2007.

[10] P. K. Choudhury, K. K. Dey, and S. Basu, "Micro- and nanoscale technologies-avenues and applications in certain directions," in Nanoscale Spectroscopy With Applications, S. M. Musa, Ed., pp. 229-266, CRC Press, 2013.

[11] S. Tretyakov, I. Nefedov, A. Sihvola, S. Maslovski, and C. Simovski, "Waves and energy in chiral nihility," Journal of Electromagnetic Waves and Applications, vol. 17, no. 5, pp. 695706, 2003.

[12] A. Lakhtakia, "An electromagnetic trinity from 'negative permittivity' and 'negative permeability"' International Journal of Infrared and Millimeter Waves, vol. 22, no. 12, pp. 1731-1734, 2001.

[13] A. Lakhtakia, "On perfect lenses and nihility," International Journal of Infrared and Millimeter Waves, vol. 23, no. 3, pp. 339343, 2002.

[14] M. A. Baqir and P. K. Choudhury, "Propagation through uniaxial anisotropic chiral waveguide under DB-boundary conditions," Journal of Electromagnetic Waves and Applications, vol. 27, no. 6, pp. 783-793, 2013.

[15] A. H. Gevorgyan and M. S. Rafayelyan, "Optics of anisotropic metamaterial based structurally chiral photonic crystals," Journal of Optics, vol. 15, no. 12, Article ID 125103, 2013.
[16] K. Y. Lim, P. K. Choudhury, and Z. Yusoff, "Chirofibers with helical windings—an analytical investigation," Optik, vol. 121, no. 11, pp. 980-987, 2010.

[17] D. Kumar, P. K. Choudhury, and F. A. Rahman, "Low eccentricity elliptical fibers with helical windings under slow-wave consideration-some special cases," Optik, vol. 121, no. 10, pp. 926-933, 2010.

[18] M. Ghasemi and P. K. Choudhury, "On the sustainment of optical power in twisted clad dielectric cylindrical fibers," Journal of Electromagnetic Waves and Applications, vol. 27, no. 11, pp. 1382-1391, 2013.

[19] M. Ghasemi and P. K. Choudhury, "Propagation through complex structured liquid crystal optical fibers," Journal of Nanophotonics, vol. 8, no. 1, Article ID 083997, 2014.

[20] M. A. Baqir and P. K. Choudhury, "Flux density through guide with microstructured twisted clad DB medium," Journal of Nanomaterials, vol. 2014, Article ID 629651, 6 pages, 2014.

[21] J. R. Pierce, Travelling Wave Tubes, D. Van Nostrand, 1950.

[22] J. Dong, "Exotic characteristics of power propagation in the chiral nihility fiber," Progress in Electromagnetics Research, vol. 99, pp. 163-178, 2009.

[23] A. H. Cherin, An Introduction To Optical Fibers, McGraw-Hill, New York, NY, USA, 1987. 

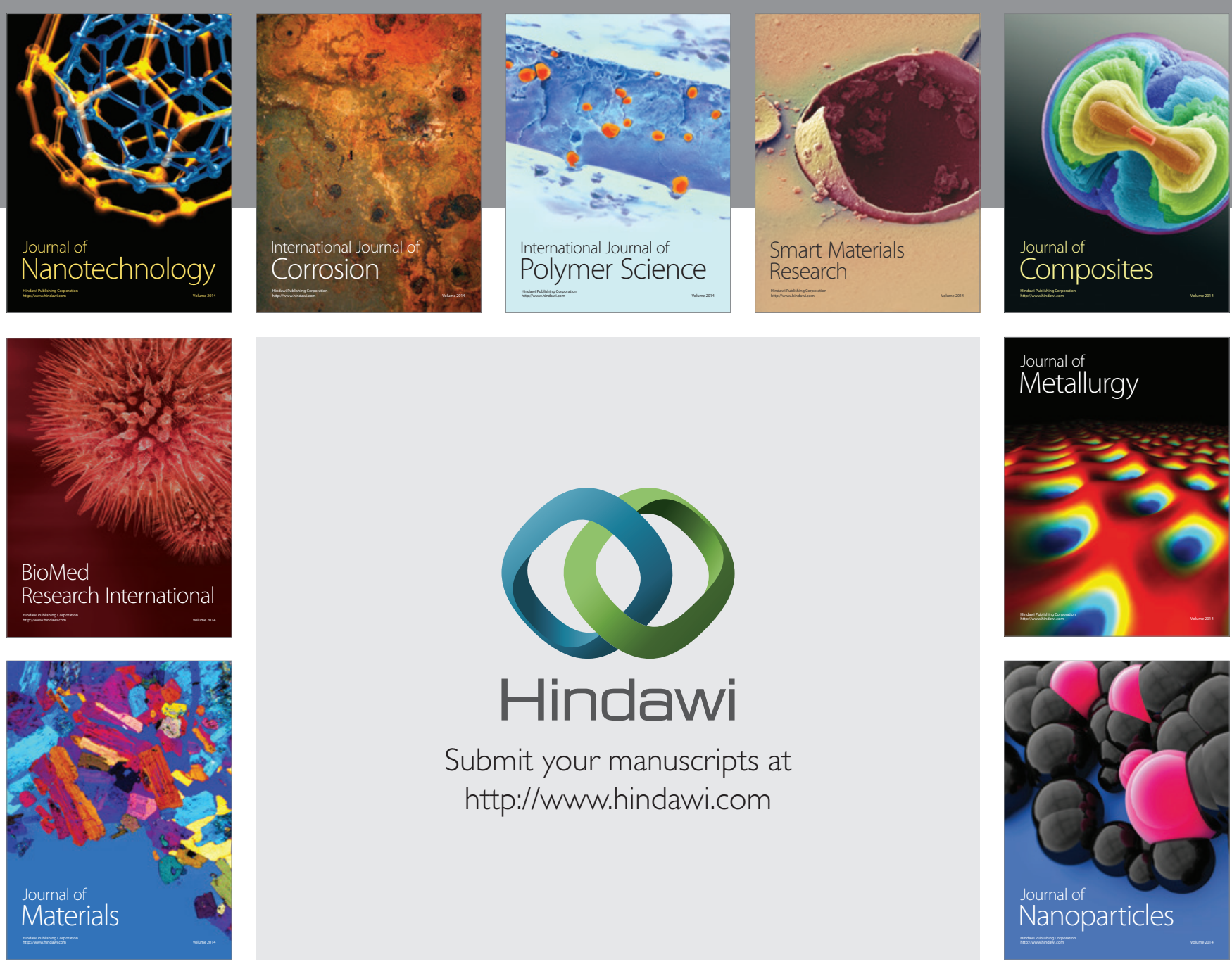

Submit your manuscripts at http://www.hindawi.com
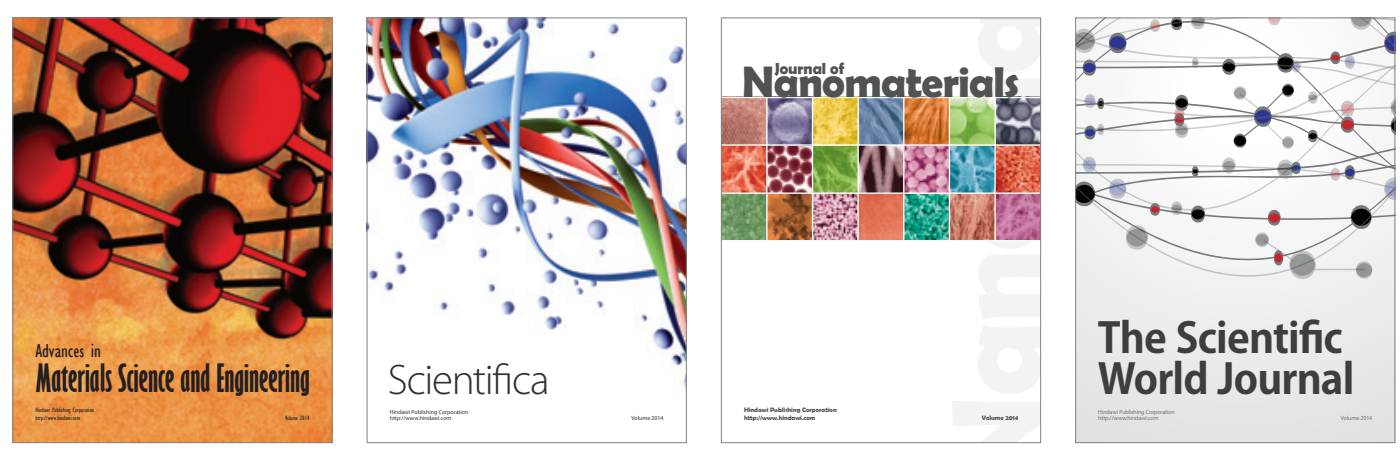

\section{The Scientific World Journal}
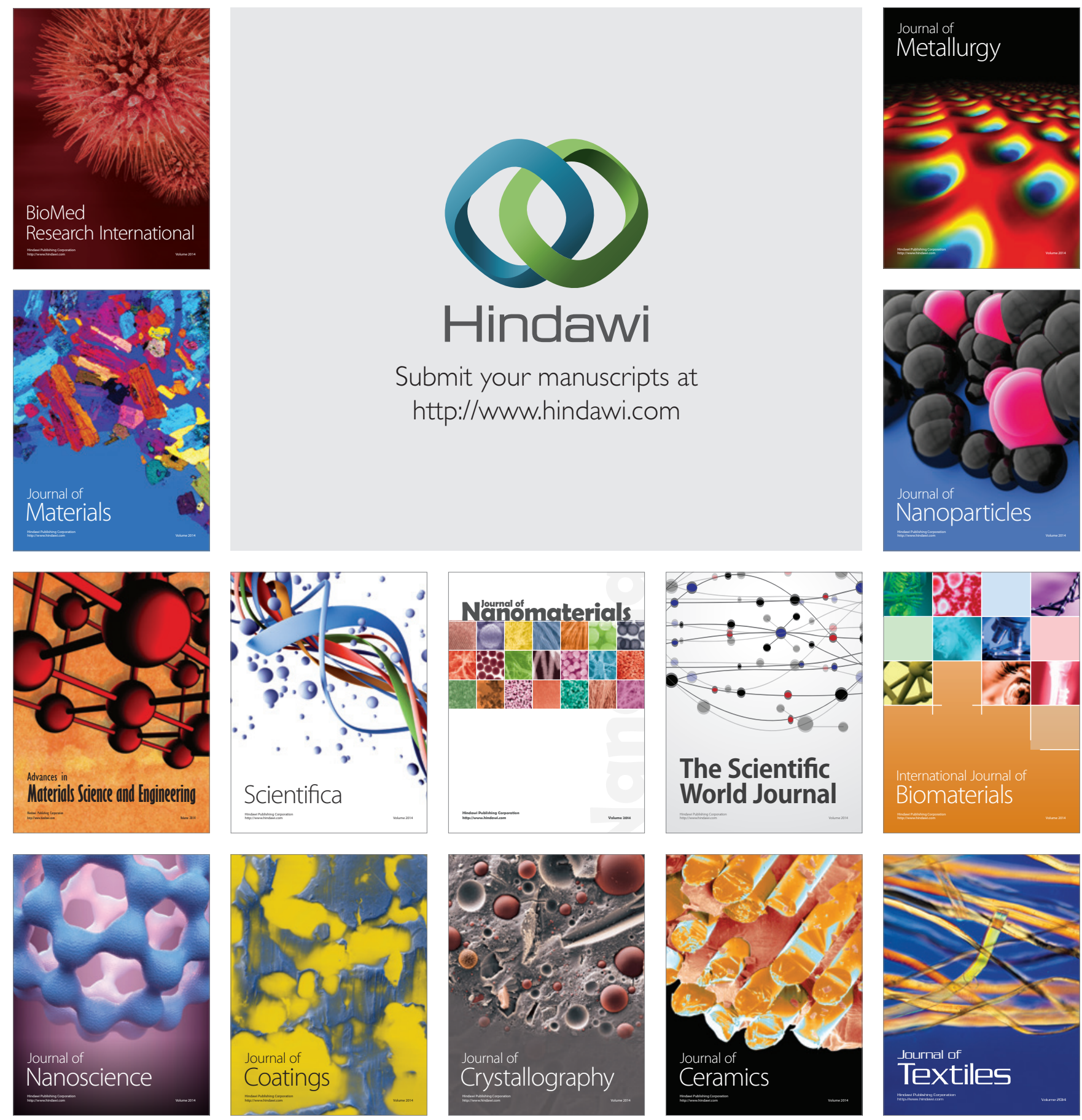